\title{
RANDERS MANIFOLDS OF POSITIVE CONSTANT CURVATURE
}

\author{
AUREL BEJANCU and HANI REDA FARRAN
}

Received 7 November 2001

\begin{abstract}
We prove that any simply connected and complete Riemannian manifold, on which a Randers metric of positive constant flag curvature exists, must be diffeomorphic to an odd-dimensional sphere, provided a certain 1-form vanishes on it.
\end{abstract}

2000 Mathematics Subject Classification: 53C60, 53C25.

1. Introduction. The geometry of Finsler manifolds of constant flag curvature is one of the fundamental subjects in Finsler geometry. Akbar-Zadeh [1] proved that, under some conditions on the growth of the Cartan tensor, a Finsler manifold of constant flag curvature $K$ is locally Minkowskian if $K=0$ and Riemannian if $K=-1$. So far, the case $K>0$ is the least understood. Bryant [9] has constructed interesting Finsler metrics of positive constant flag curvature on the sphere $S^{2}$. Recently, Bao and Shen [5] constructed nonprojectively flat Randers metrics of constant flag curvature $K>1$ on the sphere $S^{3}$. The present authors have extended the Bao-Shen result to higher dimensions (cf. Bejancu and Farran [7]). We proved that, for any constant $K>0$, there exists a Randers metric on the tangent bundle of the unit sphere $S^{2 n+1}, n \geq 1$, such that the Finsler manifold $\mathbb{F}^{2 n+1}=\left(S^{2 n+1}, F\right)$ has constant flag curvature $K$ and is not projectively flat. Recently, Shen $[13,14]$ constructed interesting examples of Randers manifolds of constant curvature, and Bao and Robles [4] found necessary and sufficient conditions for a Randers manifold to have constant flag curvature. The purpose of the present paper is to show that, subject to some natural conditions, Randers manifolds of positive constant flag curvature are diffeomorphic to odd-dimensional spheres. More precisely, we prove Theorem 2.2.

The proof we give to this theorem reveals a surprising relationship between Randers manifolds of positive constant flag curvature and Sasakian space forms.

2. Finsler manifolds of constant flag curvature. In the first part of this section, we present the concept of Finsler manifold of constant flag curvature. Then, we consider Randers manifolds and present the Yasuda-Shimada theorem [17] on Randers manifolds of positive constant curvature. Finally, we state the main result of the paper. 
Throughout the paper, we denote by $\mathscr{F}(M)$ the algebra of differentiable functions on $M$ and by $\Gamma(E)$ the $\mathscr{F}(M)$-module of the sections of a vector bundle $E$ over $M$. Also, we make use of Einstein convention, that is, repeated indices with one upper index and one lower index denote summation over their range.

Let $\mathbb{F}^{m}=(M, F)$ be a Finsler manifold, where $M$ is an $m$-dimensional $C^{\infty}$ manifold and $F$ is the Finsler metric of $\mathbb{F}^{m}$. Here, $F$ is supposed to be a $C^{\infty}$ function on the slit tangent bundle $T M^{0}=T M \backslash\{0\}$ satisfying the following conditions:

(i) $F(x, k y)=k F(x, y)$, for any $x \in M, y \in T_{x} M$, and $k>0$;

(ii) the $m \times m$ Hessian matrix

$$
\left[g_{i j}(x, y)\right]=\left[\frac{1}{2} \frac{\partial^{2} F^{2}}{\partial y^{i} \partial y^{j}}\right]
$$

is positive definite at every point $(x, y)$ of $T M^{0}$.

We denote by $\left(x^{i}, y^{i}\right)$ the coordinates on $T M^{0}$, where $\left(x^{i}\right)$ are the coordinates on $M$. The local frame field on $T M^{0}$ is $\left\{\partial / \partial x^{i}, \partial / \partial y^{i}\right\}$. Then, the Liouville vector field $L=y^{i}\left(\partial / \partial y^{i}\right)$ is a global section of the vertical vector bundle $V T M^{0}$. Moreover, $\ell=(1 / F) L$ is a unit Finsler vector field, that is, we have

$$
g_{i j}(x, y) \ell^{i} \ell^{j}=1, \quad \text { where } \ell^{i}=\frac{y^{i}}{F} .
$$

A complementary vector bundle to $V T M^{0}$ in $T T M^{0}$ is called a nonlinear connection. The canonical nonlinear connection of $\mathbb{F}^{m}$ is the distribution $G T M^{0}$ whose local frame field is given by (see Bejancu and Farran [6, page 37])

$$
\frac{\delta}{\delta x^{i}}=\frac{\partial}{\partial x^{i}}-G_{i}^{j} \frac{\partial}{\partial y^{j}},
$$

where we set

$$
G_{i}^{j}=\frac{\partial G^{j}}{\partial y^{i}} ; \quad G^{j}=\frac{1}{4} g^{j h}\left(\frac{\partial^{2} F^{2}}{\partial y^{h} \partial x^{k}} y^{k}-\frac{\partial F^{2}}{\partial x^{h}}\right) .
$$

The local coefficients $G_{i}^{j}$ are used to define the following Finsler tensor fields:

$$
R_{j}^{k}=\ell^{h}\left(\frac{\delta}{\delta x^{i}}\left(\frac{G_{h}^{k}}{F}\right)-\frac{\delta}{\delta x^{h}}\left(\frac{G_{j}^{k}}{F}\right)\right) ; \quad R_{i j}=g_{i k} R_{j}^{k}
$$

Next, we consider a flag $\ell \wedge V$ at $x \in M$ determined by $\ell$ and the tangent vector $V=V^{i}\left(\partial / \partial x^{i}\right)$. Then, according to Bao et al. [3, page 69], the flag curvature for the flag $\ell \wedge V$ is the number

$$
K(\ell, V)=\frac{V^{i} R_{i j} V^{j}}{g_{i j} V^{i} V^{j}-\left(g_{i j} \ell^{i} V^{j}\right)^{2}} .
$$


In case $K(\ell, V)$ has no dependence on $\left(x^{i}, y^{i}, V^{i}\right), i \in\{1, \ldots, m\}$, that is, $K(\ell, V)$ is a constant function, we say that $\mathbb{F}^{m}$ is a Finsler manifold of constant flag curvature. It is proved that $\mathbb{F}^{m}$ is of constant flag curvature $K$ if and only if (cf. Bao et al. [3, page 313])

$$
R_{i j}=K h_{i j}
$$

where $h_{i j}$ are the local components of the angular metric on $\mathbb{F}^{m}$ given by

$$
h_{i j}=g_{i j}-\ell_{i} \ell_{j}, \quad \text { where } \ell_{i}=g_{i j} \ell^{j} .
$$

A special Finsler metric was considered by Randers [12]. To define it, we suppose that $M$ is an $m$-dimensional manifold endowed with a Riemannian metric $\mathbf{a}=\left(a_{i j}(x)\right)$ and a nowhere zero 1-form $\mathbf{b}=\left(b_{i}(x)\right)$. Then, we define on $T M^{0}$ the function

$$
F(x, y)=\sqrt{a_{i j}(x) y^{i} y^{j}}+b_{i}(x) y^{i} .
$$

It is proved that $F$ is positive-valued on the whole $T M^{0}$ if and only if the length $\|\mathbf{b}\|$ of b satisfies (see Antonelli et al. [2, page 43])

$$
\|\mathbf{b}\|^{2}=b_{i}(x) b^{i}(x)<1
$$

where $b^{i}(x)=a^{i j}(x) b_{j}(x)$, and $\left[a^{i j}(x)\right]$ is the inverse matrix of $\left[a_{i j}(x)\right]$. A Finsler metric given by (2.9) is called a Randers metric, and $\mathbb{F}^{m}=\left(M, F, a_{i j}, b_{i}\right)$ is called a Randers manifold. Next, we consider the 1-form

$$
\beta=b^{j}\left(b_{j \mid i}-b_{i \mid j}\right) d x^{i},
$$

where the covariant derivative is taken with respect to Levi-Civita connection on $M$. In dimensions 2 and 3, Shen [13, 14] constructed examples of Randers manifolds whose flag curvature is constant and $\beta \neq 0$ on $M$. This motivated Bao and Robles [4] to determine necessary and sufficient conditions for a Randers manifold to have constant flag curvature. Also they proved that YasudaShimada theorem [17] is true with the additional condition $\beta=0$ on $M$. From these papers, we need the following result.

Theorem 2.1 (Yasuda and Shimada [17], Bao and Robles [4]). Let $\mathbb{F}^{m}=$ $\left(M, F, a_{i j}, b_{i}\right)$ be a Randers manifold such that $\beta=0$ on $M$. Then $\mathbb{F}^{m}$ is of positive constant curvature $K$ if and only if the following conditions are satisfied:

(i) the length $\|\mathbf{b}\|$ of $\mathbf{b}$ is a constant on $M$;

(ii) the covariant derivative of $\mathbf{b}$ with respect to Levi-Civita connection defined by a on $M$ satisfies

$$
b_{i \mid j}+b_{j \mid i}=0
$$


(iii) the curvature tensor of the Riemannian manifold $(M, \mathbf{a})$ is given by

$$
\begin{aligned}
R_{h i j k}= & K\left(1-\|\mathbf{b}\|^{2}\right)\left(a_{h j} a_{i k}-a_{h k} a_{i j}\right) \\
& +K\left(b_{i} b_{k} a_{h j}+b_{h} b_{j} a_{i k}-b_{i} b_{j} a_{h k}-b_{h} b_{k} a_{i j}\right) \\
& -b_{h \mid j} b_{i \mid k}+b_{h \mid k} b_{i \mid j}+2 b_{h \mid i} b_{k \mid j} .
\end{aligned}
$$

We should note that the above local components $R_{h i j k}$ are taken as follows

$$
R_{h i j k}=\mathbf{a}\left(R\left(\frac{\partial}{\partial x^{k}}, \frac{\partial}{\partial x^{j}}\right) \frac{\partial}{\partial x^{h}}, \frac{\partial}{\partial x^{i}}\right),
$$

where $R$ is the curvature tensor of Levi-Civita connection $\nabla$ on $(M, \mathbf{a})$, and it is given by

$$
R(X, Y) Z=\nabla_{X} \nabla_{Y} Z-\nabla_{Y} \nabla_{X} Z-\nabla_{[X, Y]} Z
$$

for any of the vector fields $X, Y, Z$ on $M$. As any Randers manifold of dimension $m=1$ is a Riemannian manifold, from now on we consider $m>1$.

Apart from the conditions we put in Theorem 2.1, we find in Matsumoto [11] the condition

$$
b_{i|h| k}=K\left(b_{h} a_{i k}-b_{i} a_{h k}\right) .
$$

We show here that (2.16) is a consequence of conditions (i), (ii), and (iii). First, from (2.12) we deduce that $B=b^{i}(x)\left(\partial / \partial x^{i}\right)$ is a Killing vector field on $M$. Thus, we have (cf. Yano and Kon [16, page 268])

$$
R(X, B) Y=\nabla_{X} \nabla_{Y} B-\nabla_{\nabla_{X} Y} B, \quad \forall X, Y \in \Gamma(T M),
$$

which, in local coordinates, is expressed as follows:

$$
R_{h i j k} b^{j}=b_{i|h| k}
$$

Next, from (2.10) and taking into account (i), we deduce that

$$
b_{i \mid j} b^{i}=0
$$

Then, contracting $R_{h i j k}$ by $b^{j}$ and taking into account (2.12) and (2.19), we obtain

$$
R_{h i j k} b^{j}=K\left(b_{h} a_{i k}-b_{i} a_{h k}\right)
$$

Thus, from (2.18) and (2.20), it follows (2.16). 
We make use of (2.16) in the proof of Lemma 4.2, which is crucial for proving our main result which is stated as follows.

THEOREM 2.2. Let $\mathbb{F}^{m}=\left(M, F, a_{i j}, b_{i}\right)$ be an m-dimensional Randers manifold of positive constant flag curvature with $\beta=0$ on $M$. Then $m$ must be an odd number $2 n+1$. Moreover, $M$ is a Sasakian space form that is isomorphic to the sphere $S^{2 n+1}$, provided it is a simply connected and complete manifold with respect to the Riemannian metric $\mathbf{a}=\left(a_{i j}\right)$.

3. Sasakian space forms. Let $M$ be a $(2 n+1)$-dimensional differentiable manifold and $\varphi, \xi$, and $\eta$ be a tensor field of type $(1,1)$, a vector field, and a 1-form, respectively, on $M$, satisfying

$$
\begin{gathered}
\varphi^{2}=-I+\eta \otimes \xi, \\
\eta(\xi)=1,
\end{gathered}
$$

where $I$ is the identity map on $\Gamma(T M)$. Then, we say that $M$ has a $(\varphi, \xi, \eta)$ structure. It is proved that we have (cf. Blair [8, pages 20, 21])

$$
\begin{aligned}
& \varphi(\xi)=0, \\
& \eta \circ \varphi=0 .
\end{aligned}
$$

Also, there exists a Riemannian metric a on $M$ such that

$$
\mathbf{a}(\varphi X, \varphi Y)=\mathbf{a}(X, Y)-\eta(X) \eta(Y), \quad \forall X, Y \in \Gamma(T M) .
$$

Taking $Y=\xi$ in (3.3) and using (3.1b) and (3.2a), we obtain

$$
\eta(X)=\mathbf{a}(X, \xi), \quad \forall X \in \Gamma(T M)
$$

Similarly, replace $Y$ by $\varphi X$ in (3.3), and using (3.1a), (3.2b), and (3.4), we deduce that

$$
\mathbf{a}(\varphi X, X)=0, \quad \forall X \in \Gamma(T M) .
$$

The manifold $M$ endowed with a $(\varphi, \xi, \eta$,a)-structure is a Sasakian manifold if and only if the above tensor fields satisfy (cf. Blair [8, page 73])

$$
\left(\nabla_{X} \varphi\right) Y=\mathbf{a}(X, Y) \xi-\eta(Y) X, \quad \forall X, Y \in \Gamma(T M)
$$

where $\nabla$ is the Levi-Civita connection with respect to the Riemannian metric a.

The following result on the existence of Sasakian structures on Riemannian manifolds will be used later in the paper. 
THeOrem 3.1 (Hatakeyama et al. [10]). Let $(M, \mathbf{a})$ be a $(2 n+1)$-dimensional Riemannian manifold admitting a unit Killing vector field $\xi$ such that

$$
R(X, Y) \xi=\mathbf{a}(Y, \xi) X-\mathbf{a}(X, \xi) Y, \quad \forall X, Y \in \Gamma(T M),
$$

where $R$ is the curvature tensor of the Levi-Civita connection on $M$. Then, $M$ is a Sasakian manifold.

We need the local expression of (3.7). To this end, we take $X=\partial / \partial x^{j}, Y=$ $\partial / \partial x^{i}$, and $\xi=\xi^{k}\left(\partial / \partial x^{k}\right)$. Then, using (2.15) and (3.4), we deduce that (3.7) is equivalent to

$$
\xi_{|i| j}^{k}-\xi^{k}{ }_{|j| i}=\eta_{i} \delta_{j}^{k}-\eta_{j} \delta_{i}^{k}
$$

where $\eta_{i}=\eta\left(\partial / \partial x^{i}\right)$.

Next, we denote by $\{\xi\}$ the line distribution spanned by $\xi$ on $M$. Then, the orthogonal complementary distribution to $\{\xi\}$ is denoted by $\{\xi\}^{\perp}$ and is called the contact distribution on $M$. A plane section in $T_{X} M$ is called a $\varphi$-section if there exists a vector $X \in\{\xi\}_{x}^{\perp}$ such that $\{X, \varphi X\}$ is an orthonormal basis of the plane section. The sectional curvature $H(X)$, determined by the $\varphi$-section $\operatorname{span}\{X, \varphi X\}$, is called a $\varphi$-sectional curvature. Thus, we have

$$
H(X)=\mathbf{a}(R(X, \varphi X) \varphi X, X)
$$

for any unit vector $X$ in $\{\xi\}_{x}^{\perp}$. A Sasakian manifold $M$ of constant $\varphi$-sectional curvature $c$ is called a Sasakian space form, and it is denoted by $M(c)$. There are many examples of Sasakian space forms in the literature (see Blair [8, page 99]). However, here we are interested in examples of Sasakian space forms $M(c)$ with $c>-3$. It was proved by Tanno [15] that, for any $\varepsilon>0$, there exists on the unit sphere $S^{2 n+1}$ a structure of Sasakian space form of constant $\varphi$ sectional curvature $c=-3+4 / \varepsilon$. We denote this Sasakian space form structure by $S^{2 n+1}(c)$. The same author proved the following theorem.

THEOREM 3.2 (Tanno [15]). Let $M(c)$ be a $(2 n+1)$-dimensional simply connected and complete Sasakian manifold with constant $\varphi$-sectional curvature $c>-3$. Then, $M$ is isomorphic to $S^{2 n+1}(c)$.

Here, " $M$ isomorphic to $S^{2 n+1}(c)$ " means that $M$ is diffeomorphic to $S^{2 n+1}$, and the diffeomorphism maps the structure tensors on $M(c)$ into the corresponding structure tensors on $S^{2 n+1}(c)$.

4. Proof of the main result. In the present section, we prove Theorem 2.2. The proof is based on a striking similitude we discovered between Randers manifolds of positive constant flag curvature and a special class of Sasakian space forms. First, we prove the following lemma. 
LEMMA 4.1. Let $\mathbb{F}^{m}=\left(M, F^{*}, a_{i j}^{*}, b_{i}^{*}\right)$ be a Randers manifold of positive constant flag curvature $K^{*}$. Then, there exists on $T M^{0}$ a Randers metric $F=\left(a_{i j}, b_{i}\right)$ of constant flag curvature $K=1$.

Proof. First, we define on $M$ the Riemannian metric

$$
a_{i j}(x)=K^{*} a_{i j}^{*}(x),
$$

and the 1-form

$$
b_{i}(x)=\sqrt{K^{*}} b_{i}^{*}(x) .
$$

Then, the function

$$
F(x, y)=\sqrt{a_{i j}(x) y^{i} y^{j}}+b_{i}(x) y^{i}=\sqrt{K^{*}} F^{*}(x, y)
$$

is a new Randers metric on $T M^{0}$. Also, (4.3) and (2.1) imply that

$$
\begin{aligned}
& g_{i j}(x, y)=K^{*} g_{i j}^{*}(x, y), \\
& g^{i j}(x, y)=\frac{1}{K^{*}} g^{i j *}(x, y) .
\end{aligned}
$$

Next, using (2.4), (4.3), and (4.4), we deduce that $F$ and $F^{*}$ define the same canonical nonlinear connection, that is, we have $G_{i}^{j}=G_{i}^{j *}$. As a consequence, (2.5), (4.3), and (4.4a) yield

$$
R_{i j}=R_{i j}^{*}
$$

Moreover, using (2.8) for both $F$ and $F^{*}$ and taking into account (4.3) and (4.4a), we infer that

$$
h_{i j}=K^{*} h_{i j}^{*}
$$

Finally, since $F^{*}$ is a Randers metric of positive constant flag curvature $K^{*}$, by (2.7) we have

$$
R_{i j}^{*}=K^{*} h_{i j}^{*}
$$

Thus, (4.5), (4.6), and (4.7) imply that $R_{i j}=h_{i j}$, that is, $F$ is a Randers metric of constant flag curvature $K=1$.

Next, we consider a Randers manifold $\mathbb{F}^{m}=\left(M, F, a_{i j}, b_{i}\right)$ of constant flag curvature $K=1$ and $\beta=0$. Our purpose is to prove that $M$ is a Sasakian space form. To this end, we first define on $M$ a new 1-form $\eta=(1 /\|\mathbf{b}\|) \mathbf{b}$. Clearly, $\eta$ is a unit 1 -form, that is, we have

$$
a^{i j} \eta_{i} \eta_{j}=1 .
$$


Also, from (2.12), (2.13), and (2.16), we obtain

$$
\begin{gathered}
\eta_{i \mid j}+\eta_{j \mid i}=0, \\
R_{i h j k}=\left(1-\|\mathbf{b}\|^{2}\right)\left(a_{h j} a_{i k}-a_{h k} a_{i j}\right) \\
+\|\mathbf{b}\|^{2}\left(\eta_{i} \eta_{k} a_{h j}+\eta_{h} \eta_{j} a_{i k}-\eta_{i} \eta_{j} a_{h k}-\eta_{h} \eta_{k} a_{i j}\right. \\
\left.-\eta_{h \mid j} \eta_{i \mid k}+\eta_{h \mid k} \eta_{i \mid j}+2 \eta_{h \mid i} \eta_{k \mid j}\right), \\
\eta_{i|j| k}=\eta_{j} a_{i k}-\eta_{i} a_{j k},
\end{gathered}
$$

respectively, since $\|\mathbf{b}\|$ is a constant on $M$. Now, we define on $M$ the unit vector field $\xi=\xi^{i}\left(\partial / \partial x^{i}\right)$, where we set

$$
\xi^{i}=a^{i j} \eta_{j}
$$

Then, using (4.12), (4.9), and (4.11), we deduce that

$$
\begin{gathered}
a_{i k} \xi^{k}{ }_{\mid j}+a_{j k} \xi^{k}{ }_{\mid i}=0, \\
a_{i h} \xi^{h}{ }_{|j| k}=\left(a_{i k} a_{j h}-a_{j k} a_{i h}\right) \xi^{h} .
\end{gathered}
$$

The distribution $\{\xi\}^{\perp}$ that is complementary orthogonal to the line distribution $\{\xi\}$ on $M$ is called, as in the case of Sasakian manifolds, the contact distribution on $M$. It is easy to see that $X \in \Gamma\left(\{\xi\}^{\perp}\right)$ if and only if $\eta(X)=0$. Now, we prove the following important lemmas.

LEMMA 4.2. Let $\mathbb{F}^{m}=\left(M, F, a_{i j}, b_{i}\right)$ be a Randers manifold of constant flag curvature $K=1$ and $\beta=0$. Then, $m$ must be an odd number $2 n+1, n>0$.

Proof. Using the Levi-Civita connection on $M$ with respect to the Riemannian metric $\mathbf{a}=\left(a_{i j}\right)$ and the vector field $\xi$, we define on $M$ a tensor field $\varphi=\left(\varphi_{j}^{i}\right)$ where we set

$$
\varphi_{j}^{i}=-\xi^{i} \mid j
$$

Then, (4.15), (4.12), and (4.9) yield

$$
\varphi_{j}^{i} \varphi_{k}^{j}=-a^{i h} a^{j s} \eta_{j \mid h} \eta_{s \mid k}
$$

Next, from (4.8), it follows that

$$
a^{j s} \eta_{j \mid h} \eta_{s}=0
$$

Taking the covariant derivative of (4.17) and using (4.11) and (4.8), we deduce that

$$
a^{j s} \eta_{j \mid h} \eta_{s \mid k}=-a^{j s} \eta_{s} \eta_{j|h| k}=a_{h k}-\eta_{h} \eta_{k}
$$


Taking account of (4.18) in (4.16), we obtain

$$
\varphi_{j}^{i} \varphi_{k}^{j}=-\delta_{k}^{i}+\xi^{i} \eta_{k}
$$

Finally, consider $X=X^{k}\left(\partial / \partial x^{k}\right)$ from the contact distribution of $M$, and infer that

$$
\varphi_{j}^{i} \varphi_{k}^{j} X^{k}=-X^{i}
$$

since $\eta_{k} X^{k}=0$. Hence, the restriction of $\varphi$ to $\{\xi\}^{\perp}$ is an almost complex structure. Thus, the fibers of $\{\xi\}^{\perp}$ must be of even dimension $2 n$. This implies that $m=2 n+1$ with $n>0$.

LEMMA 4.3. Let $\mathbb{F}^{m}=\left(M, F, a_{i j}, b_{i}\right)$ be a Randers manifold of constant flag curvature $K=1$ and $\beta=0$. Then, $M$ is a Sasakian manifold.

Proof. First, from (4.8) and (4.13), we deduce that there exists on $M$ a Killing vector field $\xi$. Then from (4.14), we obtain

$$
\xi_{|j| k}^{i}=\delta_{k}^{i} \eta_{j}-a_{j k} \xi^{i}
$$

which implies (3.8). Hence, by Theorem 3.1, we get the assertion of our lemma.

Since $M$ is a Sasakian manifold, we may use the local expressions of some formulas from Section 3. First, we consider a unit vector field $X=X^{i}\left(\partial / \partial x^{i}\right)$ from the contact distribution of $M$. Then, from (3.4) and (3.2b), we infer that

$$
\eta_{i} X^{i}=\eta_{i} \varphi_{j}^{i} X^{j}=0
$$

Also, (3.3) and (3.5) yield

$$
a_{i j} \varphi_{k}^{i} X^{k} \varphi_{h}^{j} X^{h}=a_{i j} X^{i} X^{j}=1, \quad a_{i j} \varphi_{k}^{i} X^{k} X^{j}=0 .
$$

Finally, using (4.9), (4.12), (4.15), and (4.20), we obtain

$$
\eta_{i \mid j} X^{i} X^{j}=0, \quad \eta_{i \mid j} X^{i} \varphi_{k}^{j} X^{k}=-\eta_{j \mid i} X^{i} \varphi_{k}^{j} X^{k}=1 .
$$

Now, we prove the following theorem.

THEOREM 4.4. Let $\mathbb{F}^{m}=\left(M, F, a_{i j}, b_{i}\right)$ be a Randers manifold of constant flag curvature $K=1$ and $\beta=0$. Then, $M$ is a Sasakian space form of constant $\varphi$-sectional curvature $c \in(-3,1)$.

Proof. Let $X^{i}=X^{i}\left(\partial / \partial x^{i}\right)$ be a unit vector field from the contact distribution of $M$. Then, using (3.9) and (2.14), we deduce that

$$
H(X)=R_{h i j k} X^{i} X^{k} \varphi_{s}^{h} X^{s} \varphi_{t}^{j} X^{t} .
$$


As by Lemma 4.3, $M$ is a Sasakian manifold, we only need to prove that $H(X)$ is a constant on $M$. To this end, we replace the components of the curvature tensor from (4.10) in (4.25), and using (4.22), (4.23), and (4.24), we obtain

$$
\begin{aligned}
& H(X)=\left(1-\|\mathbf{b}\|^{2}\right)\left\{\left(a_{h j} \varphi_{s}^{h} X^{s} \varphi_{t}^{j} X^{t}\right)\left(a_{i k} X^{i} X^{k}\right)-\left(a_{h k} \varphi_{s}^{h} X^{s} X^{k}\right)\left(a_{i j} X^{i} \varphi_{t}^{j} X^{t}\right)\right\} \\
&+\|b\|^{2}\left\{\left(\eta_{i} X^{i}\right)\left(\eta_{k} X^{k}\right) a_{h j} \varphi_{s}^{h} X^{s} \varphi_{t}^{j} X^{t}+\left(\eta_{h} \varphi_{s}^{h} X^{s}\right)\left(\eta_{j} \varphi_{t}^{j} X^{t}\right) a_{i k} X^{i} X^{k}\right. \\
& \quad-\left(\eta_{i} X^{i}\right)\left(\eta_{j} \varphi_{t}^{j} X^{t}\right) a_{h k} \varphi_{s}^{h} X^{s} X^{k}-\left(\eta_{h} \varphi_{s}^{h} X^{s}\right)\left(\eta_{k} X^{k}\right) a_{i j} X^{i} \varphi_{t}^{j} X^{t} \\
& \quad-\left(\eta_{h \mid j} \varphi_{s}^{h} X^{s} \varphi_{t}^{j} X^{t}\right)\left(\eta_{i \mid k} X^{i} X^{k}\right)+\left(\eta_{h \mid k} \varphi_{s}^{h} X^{s} X^{k}\right)\left(\eta_{i \mid j} X^{i} \varphi_{t}^{j} X^{t}\right) \\
&\left.\quad+2\left(\eta_{h \mid i} \varphi_{s}^{h} X^{s} X^{i}\right)\left(\eta_{k \mid j} X^{k} \varphi_{t}^{j} X^{t}\right)\right\} \\
&=\left(1-\|\mathbf{b}\|^{2}\right)-3\|\mathbf{b}\|^{2} \\
&=1-4\|\mathbf{b}\|^{2} .
\end{aligned}
$$

By assertion (i) of Theorem 2.1, $\|\mathbf{b}\|$ is a constant on $M$. So, $M$ is a Sasakian space form of constant $\varphi$-sectional curvature $c=1-4\|\mathbf{b}\|^{2}$. Moreover, we have $-3<c<1$ since $0<\|b\|<1$, which completes the proof of the theorem.

COROLLARY 4.5. Let $\mathbb{F}^{m}=\left(M, F^{*}, a_{i j}^{*}, b_{i}^{*}\right)$ be a Randers manifold of positive constant flag curvature $K^{*}$ and $\beta=0$. Then, $M$ is a Sasakian space form of constant $\varphi$-sectional curvature $c \in(-3,1)$.

Proof. By Lemma 4.1, there exists on $T M^{0}$ a Randers metric $F=\left(a_{i j}, b_{i}\right)$ of constant flag curvature $K=1$. Thus, the assertion of the corollary follows from Theorem 4.4.

Finally, suppose that $\mathbb{F}^{m}=\left(M, F, a_{i j}, b_{i}\right)$ is a Randers manifold satisfying the conditions from Theorem 2.2. Then, by Corollary 4.5 and Theorem 3.2, we deduce that $M$ is isomorphic to an odd-dimensional sphere. This completes the proof of our main result in Theorem 2.2.

5. Conclusions. By Theorem 2.2, we classified the simply connected and complete Randers manifolds of positive constant curvature satisfying the "BaoRobles condition" $\beta=0$. We stress that the 1 -form $b=\left(b_{i}(x)\right)$ that defines our Randers metric is nowhere zero on the manifold. Examples of Randers metrics of positive constant curvature for which $b$ vanishes at some points of the manifold are given by Shen [14], and Bao and Robles [4]. Finally, we conjecture that Randers metrics of positive constant curvature whose $\beta$ is nowhere zero on the manifold live only on open sets of $\mathbb{R}^{m}$.

\section{REFERENCES}

[1] H. Akbar-Zadeh, Sur les espaces de Finsler à courbures sectionnelles constantes [On Finsler spaces with constant sectional curvature], Acad. Roy. Belg. Bull. Cl. Sci. (5) 74 (1988), no. 10, 281-322 (French). 
[2] P. L. Antonelli, R. S. Ingarden, and M. Matsumoto, The Theory of Sprays and Finsler Spaces with Applications in Physics and Biology, Fundamental Theories of Physics, vol. 58, Kluwer Academic Publishers, Dordrecht, 1993.

[3] D. Bao, S.-S. Chern, and Z. Shen, An Introduction to Riemann-Finsler Geometry, Graduate Texts in Mathematics, vol. 200, Springer-Verlag, New York, 2000.

[4] D. Bao and C. Robles, On Randers metrics of constant curvature, preprint, 2001.

[5] D. Bao and Z. Shen, Finsler metrics of constant positive curvature on the Lie group $S^{3}$, J. London Math. Soc. (2) 66 (2002), no. 2, 453-467.

[6] A. Bejancu and H. R. Farran, Geometry of Pseudo-Finsler Submanifolds, Kluwer Academic Publishers, Dordrecht, 2000.

[7] _ Finsler metrics of positive constant flag curvature on Sasakian space forms, Hokkaido Math. J. 31 (2002), no. 2, 459-468.

[8] D. E. Blair, Contact Manifolds in Riemannian Geometry, Lecture Notes in Mathematics, vol. 509, Springer-Verlag, Berlin, 1976.

[9] R. L. Bryant, Finsler structures on the 2-sphere satisfying $K=1$, Finsler Geometry (Seattle, Wash, 1995), Contemporary Mathematics, vol. 196, American Mathematical Society, Rhode Island, 1996, pp. 27-41.

[10] Y. Hatakeyama, Y. Ogawa, and S. Tanno, Some properties of manifolds with contact metric structure, Tôhoku Math. J. (2) 15 (1963), 42-48.

[11] M. Matsumoto, Randers spaces of constant curvature, Rep. Math. Phys. 28 (1989), no. 2, 249-261.

[12] G. Randers, On an asymmetrical metric in the fourspace of general relativity, Phys. Rev. (2) 59 (1941), 195-199.

[13] Z. Shen, Finsler metrics with $K=0$ and $S=0$, preprint, 2001.

[14]_, Two-dimensional Finsler metrics with constant curvature, preprint, 2001.

[15] S. Tanno, Sasakian manifolds with constant $\phi$-holomorphic sectional curvature, Tôhoku Math. J. (2) 21 (1969), 501-507.

[16] K. Yano and M. Kon, Structures on Manifolds, Series in Pure Mathematics, vol. 3, World Scientific Publishing, Singapore, 1984.

[17] H. Yasuda and H. Shimada, On Randers spaces of scalar curvature, Rep. Mathematical Phys. 11 (1977), no. 3, 347-360.

Aurel Bejancu: Department of Mathematics and Computer Science, Kuwait University, P.O. Box 5969, Safat 13060, Kuwait

E-mail address: bejancu@mcs. sci . kuniv. edu. kw

Hani Reda Farran: Department of Mathematics and Computer Science, Kuwait University, P.O. Box 5969, Safat 13060, Kuwait

E-mail address: farran@mcs.sci. kuniv.edu.kw 


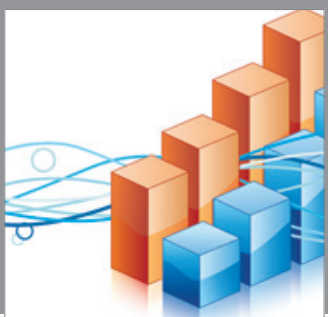

Advances in

Operations Research

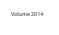

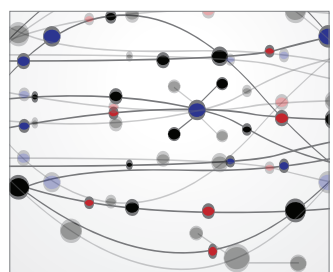

\section{The Scientific} World Journal
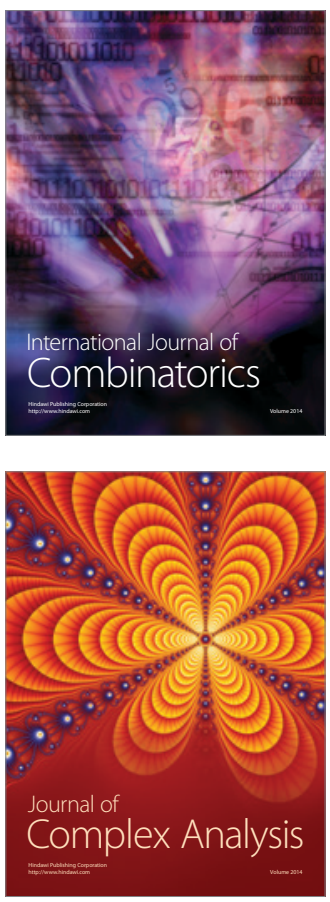

International Journal of

Mathematics and

Mathematical

Sciences
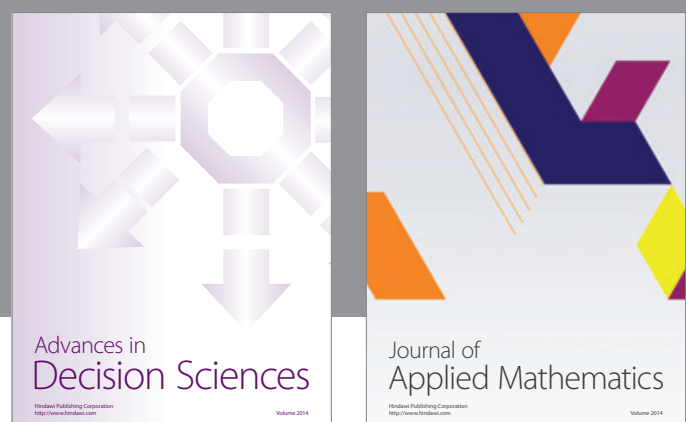

Journal of

Applied Mathematics
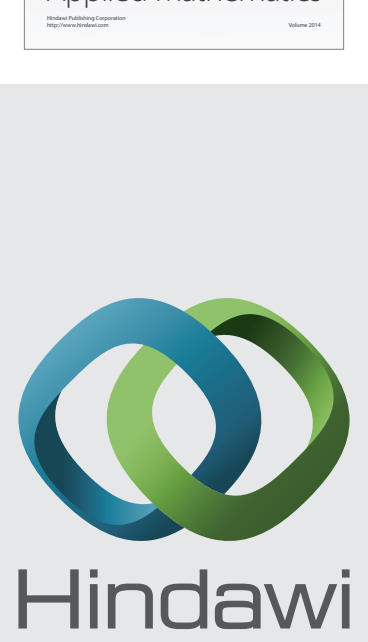

Submit your manuscripts at http://www.hindawi.com
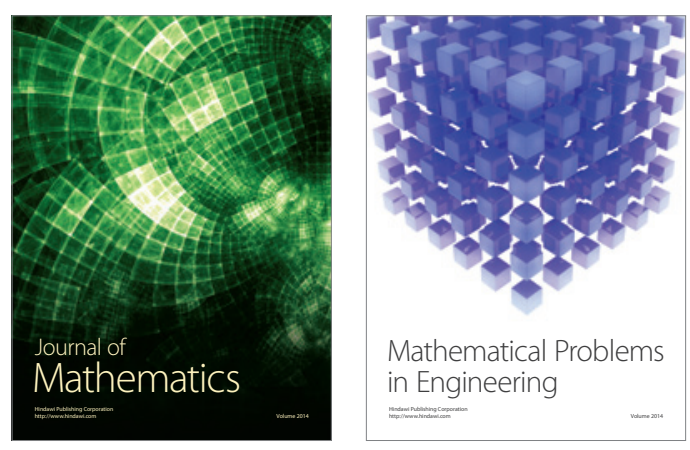

Mathematical Problems in Engineering
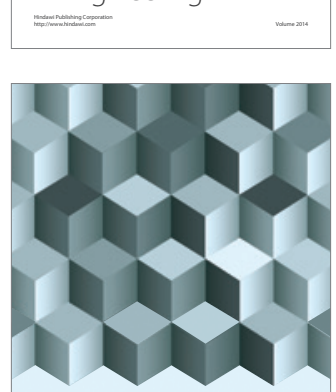

Journal of

Function Spaces
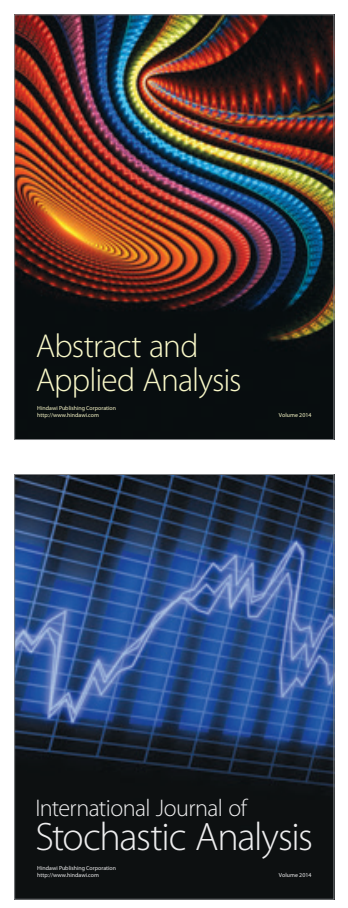

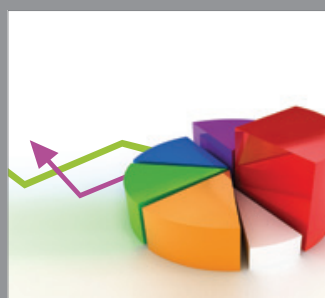

ournal of

Probability and Statistics

Promensencen
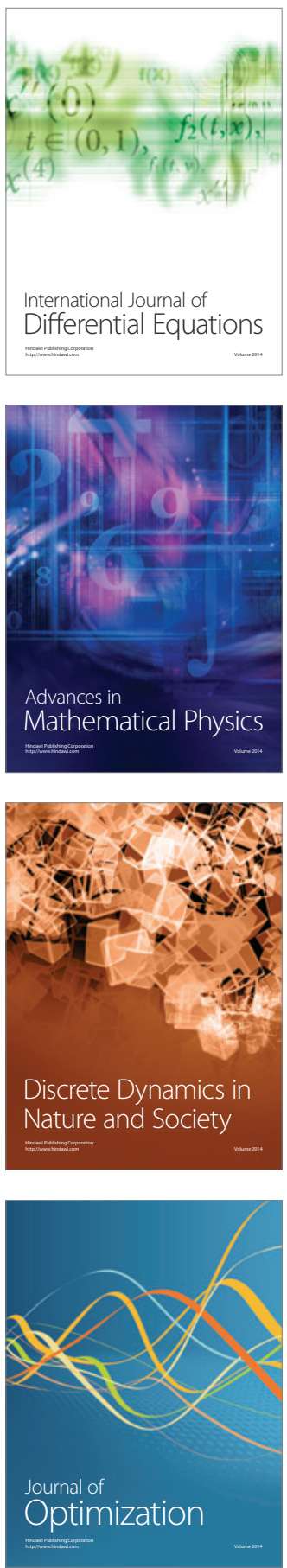